UDC 539.17

DOI: $10.22363 / 2312-9735-2018-26-4-343-356$

\title{
MAPLE program for modelling hydrogen-like atoms in quantum mechanics with non-negative distribution function
}

\author{
Alexander V. Zorin*, Nikolay P. Tretyakov ${ }^{\dagger \ddagger}$ \\ * Peoples' Friendship University of Russia (RUDN University) \\ 6 Miklukho-Maklaya str., Moscow, 117198, Russian Federation \\ ${ }^{\dagger}$ Department of Applied Information Technologies \\ The Russian Presidential Academy of National Economy and Public Administration \\ Prospect Vernadskogo, 82, Moscow 119571, Russian Federation \\ $¥$ Department of Mathematics and Information Technologies \\ Russian State Social University \\ Wilhelm Pieck str., 4-1, Moscow, 119571, Russian Federation
}

The program is proposed for a realization of the symbolic algorithm based on the quantum mechanics with non-negative probability distribution function (QDF) and for calculations of energy levels for hydrogen-like atoms. The program is written up in the language MAPLE. In the framework of the algorithm an original Maple package for calculations of necessary functions, such as hydrogen wave functions, Sturmian functions and their Fourier-transforms, Clebsch-Gordan coefficients, etc. is proposed. Operators of observables are calculated on the basis of the QDF quantization rule. According to the Ritz method, eigenvalues of Ritz matrices represent spectral values of the quantity under investigation, i.e. energy. As an example, energy levels of hydrogen-like atoms are calculated and compared with experimental data retrieved from the NIST Atomic Spectra Database Levels Data. It turns out that this theory seems to be equivalent to the traditional quantum mechanics in regard to predictions of experimental values. However, the existence of a phase-space probabilistic quantum theory may be an important advance towards the explanation and interpretation of quantum mechanics.

Key words and phrases: quantum mechanics, transition probability, computer algebra, non-negative quantum distribution function

\section{Introduction}

In [1] several computational techniques for investigation of characteristics of atomic structures were proposed. This method goes far beyond the techniques described in [2]. A deficiency of the single-configurational approximation was noted in [3]. D. Layzer [4] suggested the use of multy-configurational approximation restricting to the case of configurations belonging to the same complex (i.e. with the same parities and the same principal quantum numbers). By contrast, Eissner and Nussbaumer [1] consider interactions of configurations belonging to different complexes. Nussbaumer [5] introduced some refinement of the technique for calculation of radial functions as compared with variational approaches (for instance, Thomas-Fermi method). In particular, the procedure includes an effective charge $z$ (as compared with the real one $Z$ ):

$$
Z-N_{1}<z<Z
$$

which had been introduced in [4]. Calculations of radial functions were used in calculations of oscillators strengths and transitional probabilities. It is worth noting that the convergence of calculational results to experimental data while more and more complexes are taken into account was strictly demonstrated in [5].

The electron correlation effects on the oscillator strengths for resonance transitions in alkaline earth atoms have been studied by many authors. The conventional approach

Received $27^{\text {th }}$ October, 2018. 
consists in improving Hartree-Fock-like wavefunctions by introducing configuration interaction (CI) while using the usual dipole operator for the oscillator strength. This approach was used by Burke et al. [6] for Be, Zare [7] and Weis [8] for Mg, and Friedrich and Trefftz [9] for $\mathrm{Ca}$ and $\mathrm{Ba}$.

An alternative approach was adopted in [10], which uses an effective operator in addition to the common dipole operator and in so doing keeps a rather simple structure of corresponding wave functions. This method had been previously proposed by Hameed $[11,12]$ and it works well with alkaline metals and provides slightly worse results when applied to alkaline earth elements. However, the modification of the method proposed in [10], works very well for alkaline earths as well. Relativistic corrections introduced in $[13,14]$ were included in the extension [15] of the package [5].

It is worth noting that the whole cycle of the above-mentioned investigations has been elaborated in detail by Russian authors as well. The books [16-18] present a good introduction and an excellent review of the problem. Among other things they suggest that radial one-particle functions obtained using semi-empirical potentials, are best suited for calculations of transitional probabilities.

Quantum mechanics with non-negative distribution function (QDF) is an alternative physical theory with a developed theoretical formalism [19-28]. However, the weak point in this theory lays in the fact that until the present time no truly extensive investigations of properties of real quantum systems have been conducted. This is due to the much more complex structure of the QDF theory as compared with traditional quantum mechanics. Generations of operators of observables include calculations of complex multidimensional integrals. Furthermore, this leads to rather cumbersome expressions for operators, resulting in the absence of analytical approaches to calculations of spectra. For purposes of effective computer calculations, it is necessary to combine analytical and numerical methods, i.e. creation of a package of programs mating with each other. The evidence for the capacity of the QDF theory to obtain the same results as conventional quantum mechanics would mean an important advance in the interpretation of quantum mechanics as a statistical theory.

The present work solves the analytical part of the above-mentioned problem. The MAPLE package is used to generate necessary functions, their Fourier-transforms, to calculate integrals, operators and matrices according to formulae of QDF. Besides, numerical potentialities of MAPLE are exploited as well in calculations of eigenvalues of Ritz matrices. This program has been being developed for several years by us and co-authors, starting with [27].

The computation time raises as the dimension of Ritz matrices does. On the other hand, for small dimensions of matrices, the accuracy of the computations is insufficient. The hardware (processor and memory, for example) available for the computation may restrict either the degree of accuracy or the computation time. As a result of comparisons and tests of various program variants, we came to the conclusion that the dimension of the matrices $91 \times 91$ is optimal. Thus, this article sums up the many years of work on the creation of a package of programs and poses challenges for future research.

\section{Quantum Mechanics with Non-Negative Distribution Function}

Operators in quantum mechanics with non-negative QDF are defined up to an arbitrary set of integrable functions of configuration space and time $\phi_{k}(q, t)$, normalized by the condition:

$$
\sum_{k} \int\left|\phi_{k}(q, t)\right|^{2} \mathrm{~d} q=1 .
$$


By virtue of square integrability the functions $\phi_{k}$ allow the Fourier transformation

$$
O(A) U(q, t)=(2 \pi \hbar)^{-N} \int \phi(q-\xi, p-\eta) e^{\frac{i}{\hbar}\left(q-q^{\prime}\right) p} A(\xi, \eta, t) U\left(q^{\prime}, t\right) \mathrm{d} \xi \mathrm{d} \eta \mathrm{d} p \mathrm{~d} q^{\prime}
$$

where $\hbar$ is an arbitrary action-dimensioned constant. In what follows it is convenient to introduce a new function of phase space and time, constructed on the basic set (1) in the following manner:

$$
\phi(q, p, t)=(2 \pi \hbar)^{-\frac{N}{2}} e^{-\frac{i}{\hbar} q p} \sum_{k} \phi_{k}(q, t) \tilde{\phi}_{k}^{*}(p, t) .
$$

Then the rule for construction of quantum-mechanical operators with non-negative QDF may be formulated as follows: a classical function $A(q, p, t)$ corresponds to a linear operator $O(A)$, which acts on a generic Fourier-transformable function $u(q, t)$ in the following way:

$$
O(A) U(q, t)=(2 \pi \hbar)^{-N} \int \phi(q-\xi, p-\eta) e^{\frac{i}{\hbar}\left(q-q^{\prime}\right) p} A(\xi, \eta, t) u\left(q^{\prime}, t\right) \mathrm{d} \xi \mathrm{d} \eta \mathrm{d} p \mathrm{~d} q^{\prime} .
$$

It follows that the formulae for calculation of means are in fact equivalent:

$$
\langle A\rangle=\int \psi^{*}(q, t) O(A) \psi(q, t) \mathrm{d} q
$$

and

$$
\langle A\rangle_{\rho}=\int A(q, p, t) F_{\rho}(q, p, t) \mathrm{d} q \mathrm{~d} p
$$

where the expression

$$
F_{\psi}(q, p, t)=(2 \pi \hbar)^{-N} \sum\left|\int \phi_{k}^{*}(q-\zeta, t) \phi(\zeta, t) e^{-\frac{i}{\hbar}(\zeta, p)}\right|^{2}
$$

defines the relation between wave functions and phase space probability densities.

In this work we use the so called sturmian functions as auxiliary functions 1 . The sturmian functions satisfy the equation [29]

$$
\left(-\frac{\mathrm{d}^{2}}{\mathrm{~d} r^{2}}+\frac{l(l+1)}{r^{2}}+a_{n l} V(r)-E_{0}\right) S_{n l}(r)=0,
$$

with the Coulomb potential $V(r)=-2 / r$ and with boundary conditions $S_{n l}(0)=$ $0, S_{n l}(\infty)=0$. Here $n=1,2,3, \ldots ; l=0,1, \ldots, n-1 ; E_{0}<0 ; a_{n l}=k n ; k=\sqrt{-E_{0}}$. The solution of the equation (8) may be expressed in terms of the associated Laguerre polynomials:

$$
S_{n l}(r)=N_{n l} e^{-k r}(2 k r)^{l+1} L_{n+l}^{2 l+1}(2 k r),
$$

where $k=\sqrt{-E_{0}}$ and the normalization constant are:

$$
N_{n l}^{2}=\frac{1}{2}\left\{\frac{(n-l-1) !}{[(n+l) !]^{3}}\right\}
$$

In our package SourceFunction we use sturmian functions devided by $r$ and normalized to 1 . In the case of $n=1, l=0$, sturmian and usual Coulomb wave functions coincide 
exactly, but as $n$ grows, the amplitude and number of harmonics of sturmian functions differ from the Coulomb ones.

In the course of calculations some formulae are very useful, such as products of spherical harmonics

$$
Y_{l_{1} m_{1}}(\theta, \phi) Y_{l_{2} m_{2}}(\theta, \phi)=\sum_{L, M} \sqrt{\frac{\left(2 l_{1}+1\right)\left(2 l_{2}+1\right)}{4 \pi(2 L+1)}} C_{l_{1} 0 l_{2} 0}^{L 0} C_{l_{1} m_{1} l_{2} m_{2}}^{L M} Y_{L M}(\theta, \phi),
$$

as well as the formula for the integral of the triple product of spherical functions

$$
\begin{aligned}
\int_{0}^{2 \pi} \mathrm{d} \phi \int_{0}^{\pi} \mathrm{d} \theta Y_{l_{1} m_{1}}(\theta, \phi) Y_{l_{2} m_{2}}(\theta, \phi) Y_{l_{3} m_{3}}^{*}(\theta, \phi)= \\
\quad=\sqrt{\frac{\left(2 l_{1}+1\right)\left(2 l_{2}+1\right)}{4 \pi\left(2 l_{3}+1\right)}} C_{l_{1} 0 l_{2} 0}^{l_{3} 0} C_{l_{1} m_{1} l_{2} m_{2}}^{l_{3} m_{3}} Y_{L M}(\theta, \phi)
\end{aligned}
$$

and the expansion:

$$
\frac{1}{|\vec{q}-\vec{s}|}=4 \pi \sum_{l=0}^{\infty} \frac{1}{2 l+1} \frac{r_{-}^{l}}{r_{+}^{l+1}} \sum_{m=-l}^{l} Y_{l m}\left(\theta_{q}, \phi_{q}\right) Y_{l m}^{*}\left(\theta_{s}, \phi_{s}\right)
$$

where $r_{-}=\min (|\vec{q}|,|\vec{s}|), r_{+}=\max (|\vec{q}|,|\vec{s}|)$.

The main goal of the program is a numerical calculation of the spectrum of hydrogen and some alkali metals, using the Ritz method with $91 \times 91$ matrices. This corresponds to the use of the basis of functions with the values of the principal quantum number $n=1,2,3,4,5,6$. The obtained values are then compared with experimental data to find the optimal parameters that provide the best fit for the data. In addition, an algorithm was developed for the numerical calculation of transition probabilities for hydrogen-like atoms and a procedure for comparing these data with those obtained experimentally.

According to the second theorem of Kato, if $V(r) \rightarrow 0$ for $|\vec{r}| \rightarrow \infty$, then the essential spectrum of the operator $\hat{H}$ coincides with the essential spectrum of the operator $\hat{H}_{0}$, namely, it is half-line $R^{+}=[0, \infty)$.

The essential spectrum of an operator consists of all points of the spectrum, with the exception of isolated eigenvalues of finite multiplicity. Thus, the spectrum consists of two disjoint sets: eigenvalues of finite multiplicity and the essential spectrum.

The Hamilton operator of a hydrogen-like atom in a quantum mechanics with nonnegative distribution function is $\hat{H}=\hat{H}_{0}+c \hat{I}+V(\vec{r})$. The function $V(\vec{r})$ satisfies the conditions of the second Kato theorem. Therefore, the discrete spectrum of finite multiplicity lies below the essential spectrum and belongs to the interval $[c-1, c)$. Consequently, for the Hamilton operator $O(H)$, we apply the minimax Rayleigh-Ritz method, and the eigenvalues $\lambda_{j}^{n}$ for $N$-dimensional Ritz matrices converge to the eigenvalues $\lambda_{j}^{\infty}=\lambda_{j}$ of this operator.

The Hamiltonian operator $O(H)$ of a hydrogen-like atom in quantum mechanics with nonnegative quantum distribution function is defined by the relations

$$
O_{\left\{\phi_{k}\right\}}(H)=\hat{H}+C_{\left\{\phi_{k}\right\}} \hat{I}+V_{\left\{\phi_{k}\right\}}(\vec{r})
$$

for a set of valid functions $\left\{\phi_{k} \in L_{2}\left(R^{3}\right)\right\}$. The operator $O(H)$ is self-adjoint and bounded below by a constant $(C-1)$. The constant $C$ depends on the choice of auxiliary functions. 
In view of the nonorthogonality of the used system of coordinate functions, to find the spectrum, it is necessary to solve a generalized eigenvalue problem

$$
M \vec{x}=\lambda B \vec{x},
$$

where $M$ is the Ritz matrix $O(H)$, and $B$ is the matrix of scalar products of coordinate functions. The elements of the Ritz matrix are calculated by the formula:

$$
M_{k l}^{j}=\int \psi_{k}^{E_{0}}(\vec{r})\left[O_{j}\left(\frac{\vec{p}^{2}}{2 \mu}\right)+O_{j}\left(-\frac{Z_{\text {eff }} e^{2}}{r}\right)\right] \psi_{l}^{E_{0}} \mathrm{~d} \vec{r} .
$$

The result is a matrix that depends on the parameters $E_{0}, b_{j}, a_{j}$ and $Z_{\text {eff. }}$. These parameters were used to find the spectrum that best fits the experimental data. The calculations were carried out using the Ritz matrices of dimensions 55 and 91, which correspond to the first five or six principal quantum numbers $n$. Nevertheless, even for such limited dimensions, it was possible to obtain a spectrum of hydrogen and some alkali metals with high accuracy. To assess the quality of the model, we used the function of the quadratic discrepancy

$$
F_{\Omega}=\sum_{k \in \Omega}\left(\lambda_{k}-E_{k}^{\exp }\right)^{2} /\left(E_{k}^{\exp }\right)^{2} .
$$

As shown in our works $[22,24,25,27,28]$, for a hydrogen atom, the first 30 spectral values may be obtained with high accuracy. Moreover, the only sensitive parameter was the value $E_{0}$. For parameters $b_{j}$ in the case of a hydrogen atom, the optimal value regardless of the number of spectral values in the residual is the value $b_{j}=0$. As to the coefficients $a_{j}$, only $a_{1}=1$, and the remaining coefficients in the optimization turned to zero.

In contrast to the hydrogen atom, in the case of alkali metals it is impossible to build a single effective potential capable of describing the energy levels for different quantum numbers $n, l$. This occurs due to the interaction of the external electron with internal electrons, and the nature of this interaction is fundamentally different for different values of the orbital number $l$.

To verify the quality of the model and the correctness of the choice of auxiliary functions, transition probabilities may be calculated. In the described program, the transition probabilities are calculated by the Galerkin method with the Sturm functions of the hydrogen atom as coordinate functions, which allows us to reduce the calculations to algebraic operations with matrix elements that are calculated analytically.

\section{The Program}

The program is written up in the language MAPLE according to the algorithm described above. Figure 1 presents the general scheme of the program.

It consists of nine stages. The first stage represents the original package for calculations of necessary functions. The second stage is devoted to calculations of potential energy operators. The third stage is devoted to calculations of kinetic energy operators. The fourth stage is devoted to calculations of Ritz matrices. The fifth stage is devoted to generation of codes for Ritz matrices for later use in other programs. The sixth stage is devoted to calculation of spectra of the Hamilton operator for an electron in hydrogen-like atoms. The seventh stage is devoted to optimization of parameters of the model for hydrogen. The final stage is devoted to optimization of parameters of the model for alkaline metals. Finally, the ninth part is the calculation of transition probabilities for model verification. The structures of the parts of the software package are shown in more detail: for calculating auxiliary functions (Figure 2), for calculating matrices and energy levels (Figure 3), and for calculating transition probabilities (Figure 4). 


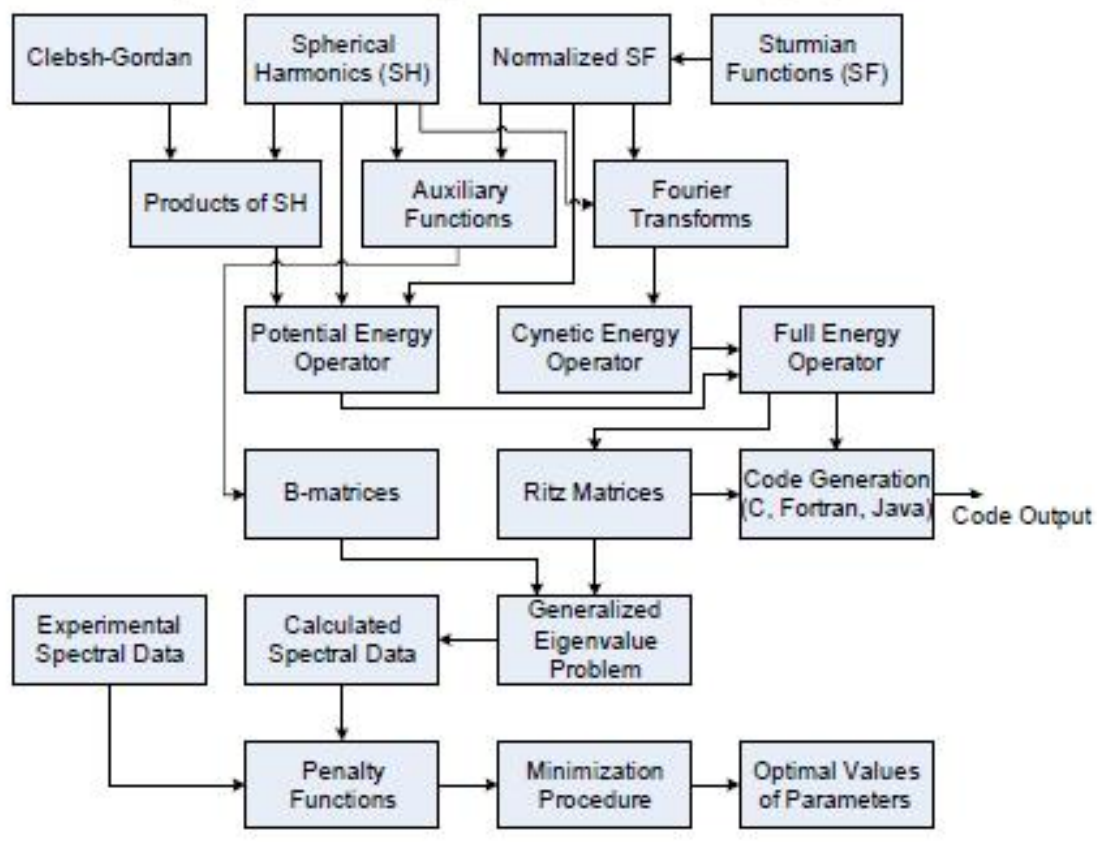

Figure 1. The global structure of the program, QDF01

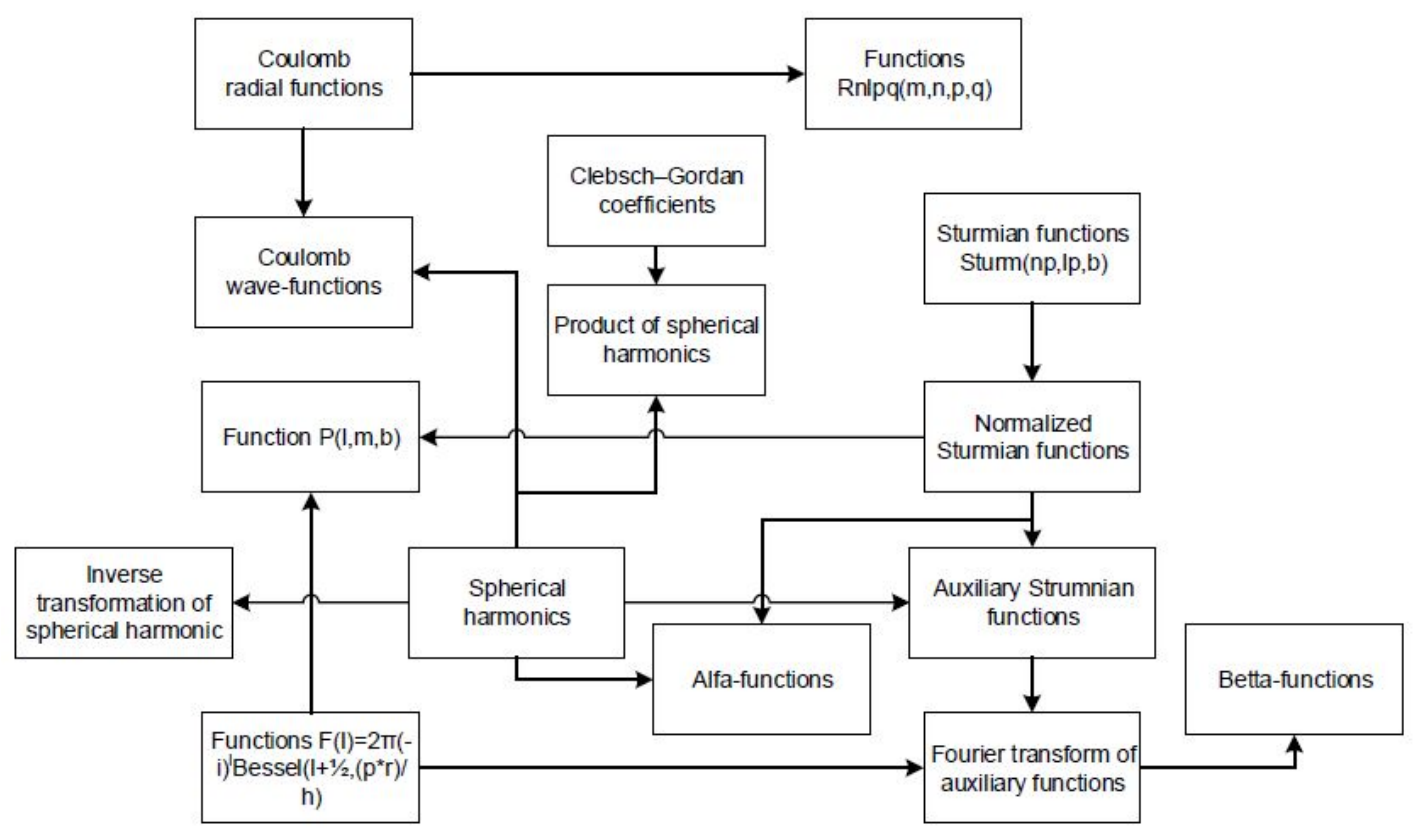

Figure 2. The structure of the part of the program for calculating auxiliary functions 


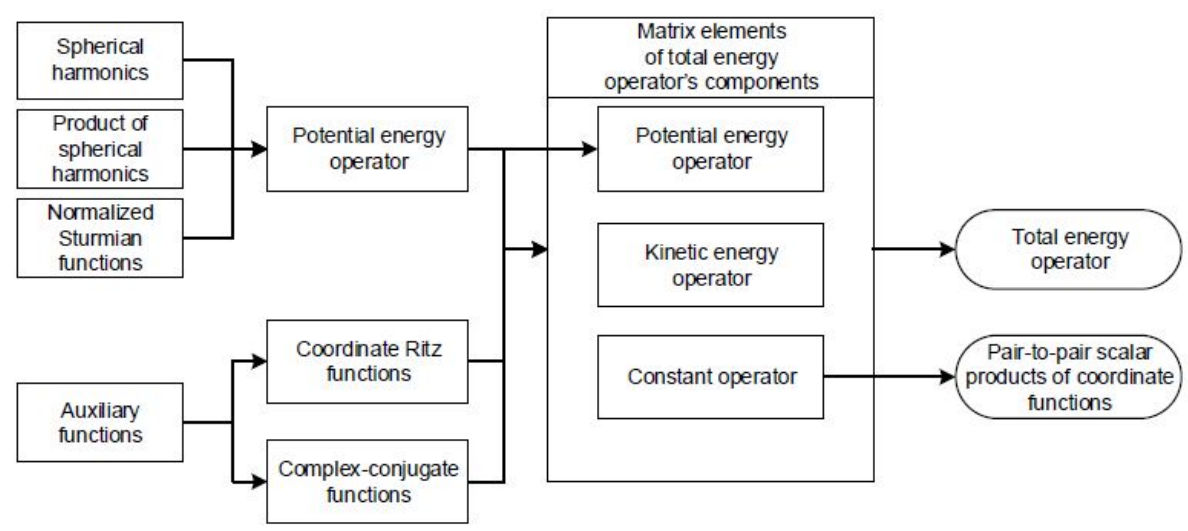

Figure 3. The structure of the part of the program for calculating matrices and energy levels

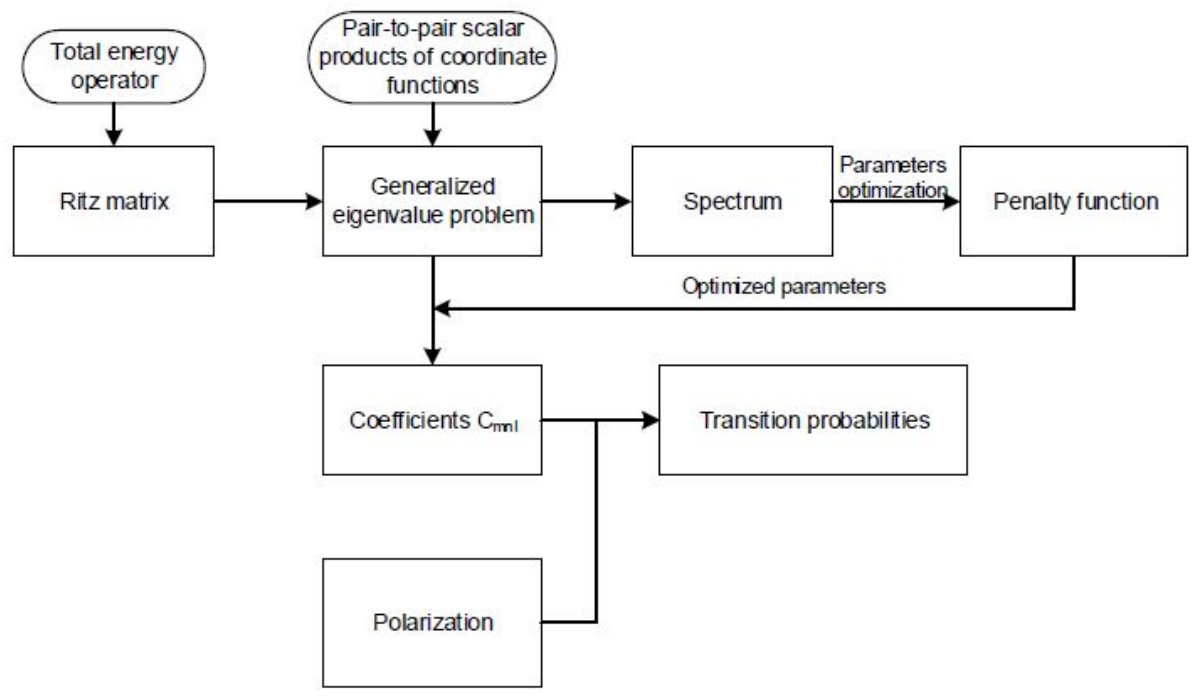

Figure 4. The structure of the part of the program for calculating transition probabilities

The subpackage "SourceFunctions" consists of the following parts. Calculation of spherical harmonics according to well-known formulae. The Legendre polynomials are generated using the Rodrigues formula. Calculations of sturmian functions, further these functions are normalized. Then follow calculations of auxiliary QDF functions (as products of sturmian functions and spherical harmonics) and calculations of fourier transforms of auxiliary functions. Let us note that all integrals are calculated analitically.

Then follow calculation of Clebsh-Gordan $S O(3)$ coefficients according to common wellknown formulae. We do not intend to carry out extensive calculations and investigations in this field, since there exist excellent Racah and Bethe packages and their extensions [30-33]. Calculations of products of spherical harmonics according to (9) etc.

Matrix elements of Ritz matrices (13) are computed and written to external files. According to the Ritz method, eigenvalues of Ritz matrices represent spectral values of 
the quantity under investigation, i.e. energy. As stated above, the algorithm consists in solving the generalized eigenvalues problem $M \vec{x}=\lambda B \vec{x}$ where $M$ is a Ritz matrix and $B$ is the matrix of pair-to-pair scalar products of coordinate functions. The program allows calculating of Ritz matrices of arbitrary dimension. However, there exist apparatus restrictions due to the degree of the dimension. Currently, calculations with dimensions 55 and 91 are available.

The parameter $E_{0}$ is adjustable. For example, Figure 5 shows the dependence of the residual on $E_{0}$ for the lithium atom and $n+l=5$.

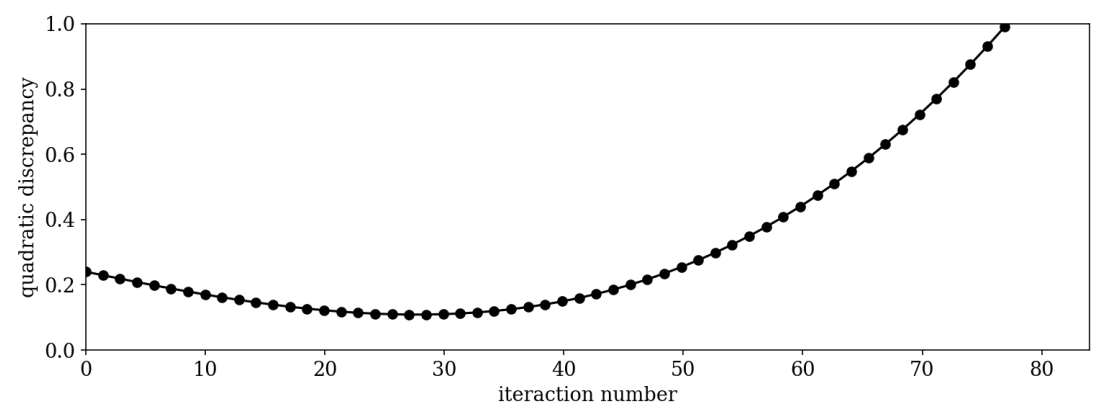

Figure 5. Graph of the quadratic discrepancy on the parameter $E_{0}$ in the case of the group of atomic levels of the lithium atom with $N+L=5$. The minimum is equal to 0.088 and corresponds to $E_{0}=-0.01414$. Other options are $b_{1}=3.33 ; b_{2}=0.001=b_{3}=b_{4}=0.001 ; Z_{\text {eff }}=1 ; m_{e}=1$

Residuals for smaller numbers of levels have similar deep minimax. But for alkali metal atoms, unlike a hydrogen atom, it is impossible to construct a unique effective potential capable of describing energy levels for different quantum numbers $n, l[27]$. One of the ways for describing energy levels in atoms of alkali metals is the so-called quantum defect model [16]. In this model, in a hydrogen-like spectrum, either the effective principal quantum number or, which is equivalent, the effective nucleus charge $Z_{\text {eff }}$ (which differs from the true charge because of the screening by the inner electrons) is used.

Figure 6 shows the dependence of the residual on the effective charge $Z_{\text {eff }}$ for the lithium atom and $N+L=5$. It is also possible to vary the effective electron mass. Figure 7 shows the dependence of the residual on the effective mass $m_{e}$ for the lithium atom and $n+l=5$.

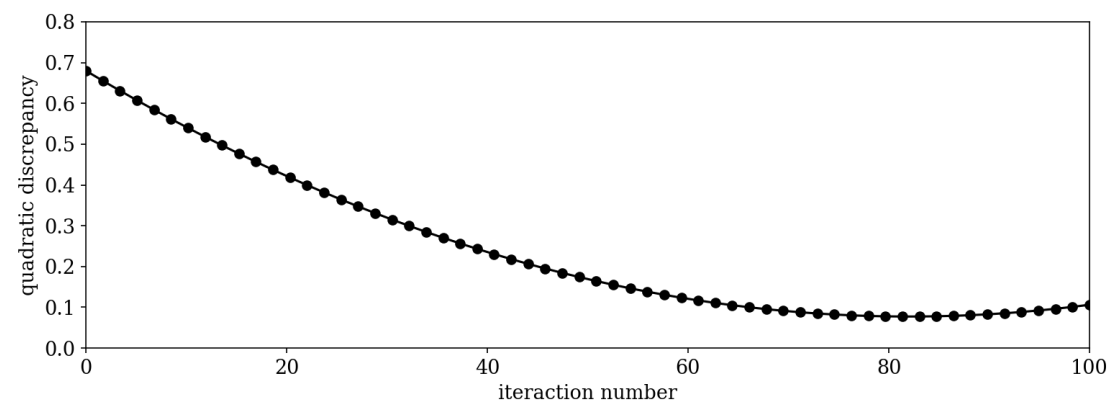

Figure 6. Graph of the quadratic discrepancy on the parameter $Z_{\text {eff }}$ in the case of the group of atomic levels of the lithium atom with $N+L=5$. The minimum is equal to 0.085 and corresponds to $Z_{\text {eff }}=-1.242$. Other options are $b_{1}=3.33 ; b_{2}=0.001=b_{3}=b_{4}=0.001 ; E_{0}=-0.1414 ; m_{e}=1$ 


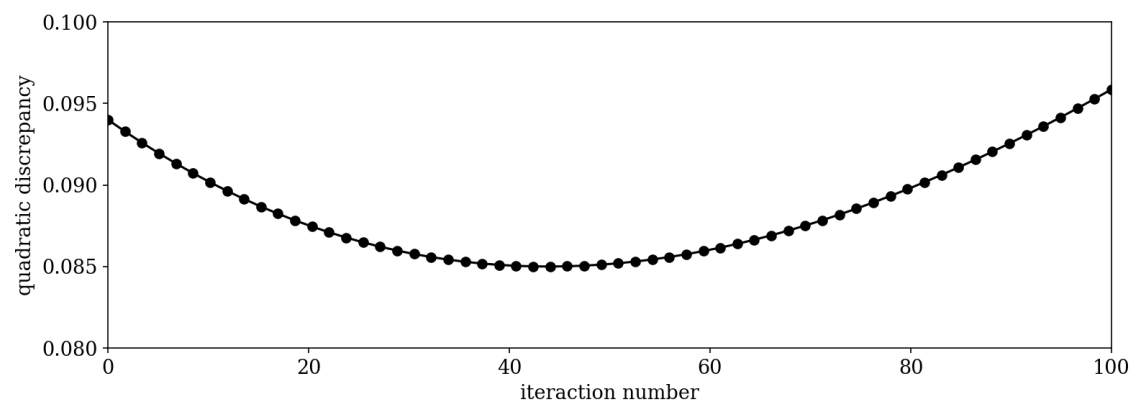

Figure 7. Graph of the quadratic discrepancy on the parameter $m_{e}$ in the case of the group of atomic levels of the lithium atom with $N+L=5$. The minimum is equal to 0.085 and corresponds to $m_{e}=0.99$. Other options are $b_{1}=3.33 ; b_{2}=0.001=b_{3}=b_{4}=0.001 ; E_{0}=-0.1414 ; Z_{\text {eff }}=1.242$

\section{Conclusion}

Quantum mechanics with a non-negative distribution function ceases to be an exotic theory and becomes a real design scheme that has its advantages. This happened after formulating it as a theory of quantum measurements. The used fitting parameters have a clear physical meaning and allow one to take into account and describe real phenomena, for example, the screening of the nuclear field by internal electrons and the interaction of an external electron with them. The proposed program allows one to effectively carry out such calculations, and also permits generalization to other quantum systems.

\section{Acknowledgments}

The publication has been prepared with the support of the "RUDN University Program 5-100" and funded by Russian Foundation for Basic Research (RFBR) according to the research project No 16-07-00556.

\section{References}

1. W. Eissner, H. Nussbaumer, Resonances in Cross Sections for Excitation of Forbidden Lines in O2+, Journal of Physics B: Atomic, Molecular and Optical Physics 2 (3) (1969) 1028-1043. doi:10.1088/0022-3700/2/3/305.

2. J. C. Slater, Atomic Shielding Constants, Physical Review 36 (1930) 57-64. doi:10.1103/PhysRev.36.57.

3. G. H. S. E. U. Condon, The Theory of Atomic Spectra, Cambridge University Press, Cambridge, 1970.

4. D. Layzer, On a Screening Theory of Atomic Spectra, Annals of Physics 8 (1959) 271-296. doi:10.1016/0003-4916(59)90023-5.

5. H. Nussbaumer, Improved Bound Wave Functions for Complex Atoms, Journal of Physics B: Atomic, Molecular and Optical Physics 5 (10) (1972) 1837-1843. doi:10.1088/0022-3700/5/10/012.

6. P. G. Burke, A. Hibbert, W. D. Robb, Wavefunctions and Oscillator Strengths of the Beryllium Iso-Electronic Sequence, Journal of Physics B: Atomic, Molecular and Optical Physics 5 (1) (1972) 37-43. doi:10.1088/0022-3700/5/1/013.

7. R. N. Zare, Correlation Effects in Complex Spectra. II. Transition Probabilities for the Magnesium Isoelectronic Sequence, Journal of Chemical Physics 47 (1967) 3561-72. doi:10.1063/1.1712423. 
8. A. W. Weiss, Theoretical multiplet strengths for Mg I, Al II, and Si III, Journal of Chemical Physics 47 (1967) 3573-3578. doi:10.1063/1.1712424.

9. H. Friedrich, E. Trefftz, Configuration Mixing and Oscillator Strengths for Some Two-Electron Spectra (Ca I, Ba I, and Others), Journal of Quantitative Spectroscopy and Radiative Transfer 9 (1969) 333-359. doi:10.1016/0022-4073(69)90030-2.

10. Y.-K. Kim, P. S. Bagus, Oscillator Strengths for the Resonance Transitions in Alkaline Earth Atoms, Journal of Physics B: Atomic, Molecular and Optical Physics 5 (10) (1972) L193-L195. doi:10.1088/0022-3700/5/10/001.

11. S. Hameed, A. Herzenberg, M. G. James, Core Polarization Corrections to Oscillator Strengths in the Alkali Atoms, Journal of Physics B: Atomic, Molecular and Optical Physics 1 (5) (1968) 822-830. doi:10.1088/0022-3700/1/5/308.

12. S. Hameed, Core Polarization Corrections to Oscillator Strengths and Singlet-Triplet Splittings in Alkaline Earth Atoms, Journal of Physics B: Atomic, Molecular and Optical Physics 9 (4) (1972) 746-760. doi:10.1088/0022-3700/5/4/009.

13. M. Jones, Relativistic Corrections to Atomic Energy Levels, Journal of Physics B: Atomic, Molecular and Optical Physics 3 (12) (1972) 1571-1592. doi:10.1088/0022$3700 / 3 / 12 / 003$.

14. M. Jones, Mutual Spin-Orbit and Spin-Spin Interactions in Atomic Structure Calculations, Journal of Physics B: Atomic, Molecular and Optical Physics 4 (11) (1971) 1422-1439. doi:10.1088/0022-3700/4/11/006.

15. W. Eissner, M. Jones, H. Nussbaumer, Techniques for the Calculation of Atomic Structures and Radiative Data Including Relativistic Corrections, Computer Physics Communications 8 (1974) 270-306. doi:10.1016/0010-4655(74)90019-8.

16. S. E. Frisch, Optical Atomic Spectrs, Fizmatgiz, Moscow, 1963, in Russian.

17. M. G. Veselov, L. N. Labzovski, Theory of Atom. Structure of Electron Scells, Nauka, Moscow, 1986, in Russian.

18. I. I. Sobelman, Atomic Spectra and Radiative Transitions, 2nd Edition, Springer, Berlin, 1996.

19. A. V. Zorin, A. L. Sevastianov, L. A. Sevastianov, Application of the Noncommutative Theory of Statistical Decisions to the Modeling of Quantum Communication Channels, IEEE, 2017, pp. 26-31. doi:10.1109/ICUMT.2017.8255195.

20. V. V. Kuryshkin, La mechanique quantique avec une function nonnegative de distribution dans l'espace des phases, Annales de l'I.H.P. Physique théorique 17 (1972) 81-95.

21. V. V. Kuryshkin, Some Problems of Quantum Mechanics Possessing a Non-Negative Phase-Space Distribution Function, International Journal of Theoretical Physics 7 (1973) 451-466. doi:10.1007/BF00713247.

22. A. V. Gorbachev, L. A. Sevastianov, A. V. Zorin, Kuryshkin-Wodkiewicz Model of Quantum Measurements for Atoms and Ions with One Valence Electron, Bulletin of Peoples' Friendship University of Russia. Series: Mathematics. Information Sciences. Physics 2 (2016) 44-52.

23. A. V. Zorin, V. V. Kuryshkin, L. A. Sevastianov, Description of the Spectrum of a Hydrogen-Like Atom, Bulletin of Peoples' Friendship University of Russia. Series: Physics 6 (1998) 62-66, in Russian.

24. A. V. Zorin, L. A. Sevastianov, Hydrogen-Like Atom with Nonnegative Quantum Distribution Function, Physics of Atomic Nuclei 70 (2007) 792-799. doi:10.1134/S1063778807040229.

25. L. Sevastianov, A. Zorin, A. Gorbachev, Pseudo-Differential Operators in the Operational Model of a Quantum Measurement of Observables, Lecture Notes in Computer Science 7152 (2012) 174-181. doi:10.1007/978-3-642-28212-6_17.

26. A. V. Zorin, The Operational Model of Quantum Measurement of KuryshkinWodkiewicz, Bulletin of Peoples' Friendship University of Russia. Series: Mathematics. Information Sciences. Physics 2 (2012) 42-54, in Russian. 
27. A. V. Zorin, L. A. Sevastianov, N. P. Tretyakov, Computer Modelling of Hydrogen-Like Atoms in Quantum Mechanics with Nonnegative Distribution Function, Programming and Computer Software 33 (2007) 94-104. doi:10.1134/S0361768807020077.

28. L. A. Sevastianov, A. V. Zorin, The Computer-Based Model of Quantum Measurement, Physics of Atomic Nuclei 80 (2017) 774-780. doi:10.1134/S1063778817040238.

29. M. Rotenberg, Theory and Application of Sturmian Functions, Advances in Atomic and Molecular Physics 6 (1970) 233-268. doi:10.1016/S0065-2199(08)60206-7.

30. K. Rykhlinskaya, S. Fritzsche, Use of Group Theory for the Analysis of Vibrational Spectra, Computer Physics Communications 162 (2004) 124-142. doi:10.1016/j.cpc.2004.06.088.

31. K. Rykhlinskaya, S. Fritzsche, Generation of Molecular Symmetry Orbitals for the Point and Double Groups, Computer Physics Communications 171 (2005) 119-132. doi:10.1016/j.cpc.2005.03.112.

32. K. Rykhlinskaya, S. Fritzsche, Generation of Clebsch-Gordan Coefficients for the Point and Double Groups, Computer Physics Communications 174 (2006) 903-913. doi:10.1016/j.cpc.2006.01.001.

33. O. Gaigalas, O. Scharf, S. Fritzsche, Maple Procedures for the Coupling of Angular Momenta. VIII. Spin-Angular Coefficients for Single-Shell Configurations, Computer Physics Communications 166 (2005) 141-169. doi:10.1016/j.cpc.2004.11.003.

УДК 539.17

DOI: $10.22363 / 2312-9735-2018-26-4-343-356$

\title{
MAPLE программа для моделирования водородоподобных атомов в квантовой механике с неотрицательной функцией распределения
}

\author{
А. В. Зорин ${ }^{*}$ Н. П. Третьяков ${ }^{\dagger \ddagger}$ \\ * Российский университет дружбъ народов \\ ул. Миклухо-Маклая, д. 6, Москва, Россия, 117198 \\ ${ }^{\dagger}$ Кафедра прикладных информачионных технологий \\ Российская академия народного хозяйства и государственной службы при Президенте \\ Российской федерачии \\ Проспект Вернадского, д. 82, Москва, Россия, 119571 \\ ‡ Кафедра математики и информатики \\ Российский государственный соииалъный университет \\ ул. Вильгелвма Пика, д. 4-1, Москва, Россия, 119571
}

\begin{abstract}
Предложена программа для реализации алгоритма аналитических вычислений, основанного на квантовой механике с неотрицательной функцией распределения вероятностей и для расчётов уровней энергии для водородоподобных атомов. Программа написана на языке MAPLE. В рамках алгоритма осуществляются вычисления необходимых функций, таких как волновые функции атома водорода, штурмовские функции и их фурье-преобразования, коэффициенты Клебша-Гордана и т. д. Операторы наблюдаемых вычисляются на основе правила квантования квантовой механики с неотрицательной функцией распределения. Согласно методу Ритца, собственные значения матриц Ритца представляют собой спектральные значения исследуемой величины, т. е. энергии. В качестве примера вычисляются энергетические уровни водородоподобных атомов и сравниваются с экспериментальными значениями, полученными из данных уровней базы данных NIST Atomic Spectra. Используемая теория, по-видимому, эквивалентна традиционной квантовой механике в отношении предсказаний экспериментальных значений. Однако существование вероятностной квантовой теории фазового пространства может быть важным шагом вперёд к объяснению и интерпретации квантовой механики.
\end{abstract}

Ключевые слова: квантовая механика, вероятность перехода, компьютерная алгебра, неотрицательная квантовая функция распределения 


\section{Литература}

1. Eissner W., Nussbaumer H. Resonances in Cross Sections for Excitation of Forbidden Lines in O2+ // Journal of Physics B: Atomic, Molecular and Optical Physics. 1969. - Vol. 2, No 3. - Pp. 1028-1043. — DOI: 10.1088/0022-3700/2/3/305.

2. Slater J. C. Atomic Shielding Constants // Physical Review. - 1930. — Vol. 36. Pp. 57-64. - DOI: 10.1103/PhysRev.36.57.

3. E. U. Condon G. H. S. The Theory of Atomic Spectra. - Cambridge: Cambridge University Press, 1970.

4. Layzer D. On a Screening Theory of Atomic Spectra // Annals of Physics. - 1959. Vol. 8, issue 2. - Pp. 271-296. - DOI: 10.1016/0003-4916(59)90023-5.

5. Nussbaumer H. Improved Bound Wave Functions for Complex Atoms // Journal of Physics B: Atomic, Molecular and Optical Physics. — 1972. — Vol. 5, No 10. Pp. 1837-1843. - DOI: 10.1088/0022-3700/5/10/012.

6. Burke P. G., Hibbert A., Robb W. D. Wavefunctions and Oscillator Strengths of the Beryllium Iso-Electronic Sequence // Journal of Physics B: Atomic, Molecular and Optical Physics. — 1972. — Vol. 5, No 1. - Pp. 37-43. — DOI: 10.1088/0022$3700 / 5 / 1 / 013$.

7. Zare R. N. Correlation Effects in Complex Spectra. II. Transition Probabilities for the Magnesium Isoelectronic Sequence // Journal of Chemical Physics. - 1967. Vol. 47, issue 9. - Pp. 3561-72. - DOI: 10.1063/1.1712423.

8. Weiss A. W. Theoretical multiplet strengths for Mg I, Al II, and Si III // Journal of Chemical Physics. - 1967. - Vol. 47, issue 9. - Pp. 3573-3578. DOI: $10.1063 / 1.1712424$.

9. Friedrich H., Trefftz E. Configuration Mixing and Oscillator Strengths for Some TwoElectron Spectra (Ca I, Ba I, and Others) // Journal of Quantitative Spectroscopy and Radiative Transfer. - 1969. - Vol. 9, issue 3. - Pp. 333-359. — DOI: 10.1016/00224073(69)90030-2.

10. Kim Y.-K., Bagus P. S. Oscillator Strengths for the Resonance Transitions in Alkaline Earth Atoms // Journal of Physics B: Atomic, Molecular and Optical Physics. 1972. - Vol. 5, No 10. — Pp. L193-L195. — DOI: 10.1088/0022-3700/5/10/001.

11. Hameed S., Herzenberg A., James M. G. Core Polarization Corrections to Oscillator Strengths in the Alkali Atoms // Journal of Physics B: Atomic, Molecular and Optical Physics. - 1968. — Vol. 1, No 5. - Pp. 822-830. — DOI: 10.1088/0022-3700/1/5/308.

12. Hameed $S$. Core Polarization Corrections to Oscillator Strengths and Singlet-Triplet Splittings in Alkaline Earth Atoms // Journal of Physics B: Atomic, Molecular and Optical Physics. — 1972. — Vol. 9, No 4. — Pp. 746-760. — DOI: 10.1088/0022$3700 / 5 / 4 / 009$.

13. Jones $M$. Relativistic Corrections to Atomic Energy Levels // Journal of Physics B: Atomic, Molecular and Optical Physics. - 1972. — Vol. 3, No 12. — Pp. 1571-1592. DOI: $10.1088 / 0022-3700 / 3 / 12 / 003$.

14. Jones M. Mutual Spin-Orbit and Spin-Spin Interactions in Atomic Structure Calculations // Journal of Physics B: Atomic, Molecular and Optical Physics. - 1971. Vol. 4, No 11. - Pp. 1422-1439. — DOI: 10.1088/0022-3700/4/11/006.

15. Eissner W., Jones M., Nussbaumer $H$. Techniques for the Calculation of Atomic Structures and Radiative Data Including Relativistic Corrections // Computer Physics Communications. — 1974. — Vol. 8, issue 4. - Pp. 270-306. — DOI: 10.1016/00104655(74)90019-8.

16. Фриш С. Э. Оптические спектры атомов. - М.: Физматгиз, 1963.

17. Веселов М. Г., Лабзовский Л. Н. Теория атома.Строение электронных оболочек. М.: Наука, 1986.

18. Sobelman I. I. Atomic Spectra and Radiative Transitions. - 2nd edition. - Berlin: Springer, 1996. 
19. Zorin A. V., Sevastianov A. L., Sevastianov L. A. Application of the Noncommutative Theory of Statistical Decisions to the Modeling of Quantum Communication Channels // 9th International Congress on Ultra Modern Telecommunications and Control Systems and Workshops (ICUMT). — IEEE, 2017. — Pp. 26-31. DOI: 10.1109/ICUMT.2017.8255195.

20. Kuryshkin $V . V$. La mechanique quantique avec une function nonnegative de distribution dans l'espace des phases // Annales de l'I.H.P. Physique théorique. - 1972 . Vol. 17. - Pp. 81-95.

21. Kuryshkin V. V. Some Problems of Quantum Mechanics Possessing a Non-Negative Phase-Space Distribution Function // International Journal of Theoretical Physics. 1973. - Vol. 7, issue 6. — Pp. 451-466. — DOI: 10.1007/BF00713247.

22. Gorbachev A. V., Sevastianov L. A., Zorin A. V. Kuryshkin-Wodkiewicz Model of Quantum Measurements for Atoms and Ions with One Valence Electron // Bulletin of Peoples' Friendship University of Russia. Series: Mathematics. Information Sciences. Physics. - 2016. - Vol. 2. - Pp. 44-52.

23. Зорин А. В., Курышкин В. В., Севастъянов Л. А. Описание спектра водородоподобного атома // Вестник РУДН. Серия: Физика. - 1998. - Т. 6. - С. 62-66.

24. Zorin A. V., Sevastianov L. A. Hydrogen-Like Atom with Nonnegative Quantum Distribution Function // Physics of Atomic Nuclei. - 2007. — Vol. 70. - Pp. 792 799. — DOI: 10.1134/S1063778807040229.

25. Sevastianov L., Zorin A., Gorbachev A. Pseudo-Differential Operators in the Operational Model of a Quantum Measurement of Observables // Lecture Notes in Computer Science. — 2012. — Vol. 7152. — Pp. 174-181. — DOI: 10.1007/978-3-642-28212-6_17.

26. Зорин A. B. Операционная модель квантовых измерений Курышкина- Вудкевича // Вестник РУДН. Серия: Математика. Информатика. Физика. - 2012. T. 2. - C. $42-54$.

27. Zorin A. V., Sevastianov L. A., Tretyakov N. P. Computer Modelling of HydrogenLike Atoms in Quantum Mechanics with Nonnegative Distribution Function // Programming and Computer Software. - 2007. - Vol. 33, issue 2. - Pp. 94-104. DOI: $10.1134 /$ S0361768807020077.

28. Sevastianov L. A., Zorin A. V. The Computer-Based Model of Quantum Measurement // Physics of Atomic Nuclei. - 2017. - Vol. 80, issue 4. - Pp. 774-780. DOI: 10.1134/S1063778817040238.

29. Rotenberg $M$. Theory and Application of Sturmian Functions // Advances in Atomic and Molecular Physics. - 1970. — Vol. 6. — Pp. 233-268. — DOI: 10.1016/S00652199(08)60206-7.

30. Rykhlinskaya K., Fritzsche S. Use of Group Theory for the Analysis of Vibrational Spectra // Computer Physics Communications. - 2004. - Vol. 162, issue 2. Pp. 124-142. — DOI: 10.1016/j.cpc.2004.06.088.

31. Rykhlinskaya K., Fritzsche S. Generation of Molecular Symmetry Orbitals for the Point and Double Groups // Computer Physics Communications. - 2005. - Vol. 171, issue 2. - Pp. 119-132. — DOI: 10.1016/j.cpc.2005.03.112.

32. Rykhlinskaya K., Fritzsche S. Generation of Clebsch-Gordan Coefficients for the Point and Double Groups // Computer Physics Communications. - 2006. - Vol. 174, issue 11. - Pp. 903-913. — DOI: 10.1016/j.cpc.2006.01.001.

33. Gaigalas O., Scharf O., Fritzsche S. Maple Procedures for the Coupling of Angular Momenta. VIII. Spin-Angular Coefficients for Single-Shell Configurations // Computer Physics Communications. - 2005. - Vol. 166, issue 2. - Pp. 141-169. DOI: $10.1016 /$ j.cpc.2004.11.003.

(C) Zorin Alexander V., Tretyakov Nikolay P., 2018

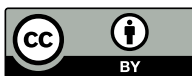


This work is licensed under a Creative Commons Attribution 4.0 International License

Для цитирования:

Zorin A.V., Tretyakov N.P. MAPLE program for modelling hydrogen-like atoms in quantum mechanics with non-negative distribution function // RUDN Journal of Mathematics, Information Sciences and Physics. — 2018. — Vol. 26, No 4. - Pp. 343-356. DOI: $10.22363 / 2312-9735-2018-26-4-343-356$.

\section{For citation:}

Zorin A. V., Tretyakov N. P. MAPLE program for modelling hydrogen-like atoms in quantum mechanics with non-negative distribution function, RUDN Journal of Mathematics, Information Sciences and Physics 26 (4) (2018) 343-356. DOI: 10.22363/2312-97352018-26-4-343-356.

Сведения об авторах:

Зорин Александр Валерьевич - доцент, кандидат физико-математических наук, доцент РУДН (е-mail: zorin@mx.rudn.ru, тел.: +7(495)9522572)

Третьяков Николай Павлович - доцент, кандидат физико-математических наук, доцент кафедры математики и информатики РГСУ (e-mail: trn110rambler.ru, тел.: +7(495)9522572)

Information about the authors:

Zorin, Alexander V. - Associate Professor, Candidate of Sciences in Physics and Mathematics, Associate Professor of Peoples' Friendship University of Russia (RUDN University) (e-mail: zorin@mx.rudn.ru, phone: +7(495)9522572)

Tretyakov, N. P. - Associate Professor, Candidate of Sciences in Physics and Mathematics, Associate Professor of Department of Mathematics and Information Technologies of Russian State Social University (e-mail: trn11@rambler.ru, phone: +7(495)9522572) 